rheuma plus $2017 \cdot 16: 1$

DOI 10.1007/s12688-017-0107-5

(c) Springer-Verlag Wien 2017
Burkhard Leeb

II. Medizinische Abteilung, NOE Kompetenzzentrum für Rheumatologie, Stockerau

\title{
Biologika, Biosimilars und small molecules
}

Liebe Leserinnen und Leser,

wir schreiben 2017 und eines erscheint sicher, die Rheumatologie bleibt spannend. Denn die Debatte um Biologika, Biosimilars und auch small molecules, von besonders Theoriegläubigen auch targeted synthetic DMARDs genannt, wird mit $\mathrm{Si}$ cherheit weitergehen; etwas Anderes ist aber, in aller Redlichkeit betrachtet, nicht wirklich vorherzusehen - der Ausgang dieses Wettstreits.

Werden die Biosimilars die Erwartungen, die vor allem die Zahler im Gesundheitswesen, die Hersteller und die Gefolgsleute der beiden in sie setzen, erfüllen? Werden Biologicals generell ihre Position an der Spitze der Therapiekaskade entzündlich-rheumatischer Erkrankungen halten, durch neue Präparate, vorwiegend Il-6 -oder Il-17 Antagonisten eventuell sogar weiter ausbauen können? Oder lösen die small molecules, derzeit ja vor allem Januskinase (JAK) -Inhibitoren, die Injektionen als Errungenschaft ab? Vergessen sollte man nicht, dass zwar der Name Januskinase sehr publikumswirksam klingt, dass aber JAK angeblich eigentlich als Synonym für „just another kinase“ vom Entdecker verwendet wurde. Möglicherweise kommen ja auch noch andere - zu der JAK-Hemmung oder Phosphodiesterase-Antagonismus alternative - Wirkprinzipien zum Tragen.

Jedenfalls wird dem Wissen über und Verständnis für Biologika-Therapien weiter ein großer Stellenwert zuzuordnen sein. Register sind in zunehmendem Maße die Datenquelle in dieser Hinsicht, erlauben sie doch eine Beurteilung der Verhältnisse, fernab von der elfenbeinernen Welt der klinischen Studien. Umso wichtiger ist es daher, die Validität der in Registern erhobenen Daten nachzuweisen. In dieser Ausgabe finden Sie eine Zusammenfassung der ersten publizierten peer-reviewten Ar- beit (Bernhard Rintelen, Jochen Zwerina et al.) aus dem österreichischen BioReg, welche die Situation in Hinblick auf Biologika-Therapie in unserem Lande beleuchtet. Dieser Untersuchung gemäß können wir Österreicher uns durchaus glücklich über die gesundheitspolitische Situation in unserem Land schätzen.

Die Rheumatologie darf aber, bei aller Bedeutung der autoimmunologisch begründeten Erkrankungen, nicht außer Acht lassen, dass die Gesamtheit der muskuloskelettalen Erkrankungen in ihr Spektrum fällt. Die Jahrestagung der Österreichischen Gesellschaft für Rheumatologie und Rehabilitation war bemüht, auch diesen Erkrankungen eine Bühne zu bieten. In diesem Heft werden Beiträge von Roland Kocijan zur Osteoporosetherapie, von Andrea Österbauer über Fibromyalgie, von Johann Gruber über die Therapie der Osteoarthritis und von Rudolf Puchner über Komorbiditäten bei Rheumatoider Arthritis diesen Aspekten der Rheumatologie Rechnung tragen.

Zweifellos spielt die Immundiagnostik von Arthritiden eine wichtige Rolle im diagnostischen Prozess, die aber nur im klinischen Kontext gesehen wird, da sie sonst auch zur Desinformation beitragen kann. Stichwort: Die wenigsten Laborwerte machen eine Menschen per se zum Patienten. Der Nachweis von Antikörpern (Ak) gegen citrullinierte Proteine hat die Immundiagnostik von Arthritiden zweifelsohne bereichert, demgegenüber spielt die Bestimmung von Zytokinkonzentrationen in Plasma oder Synovia in der Diagnostik von Arthritiden eine eher untergeordnete Rolle. Die indirekte Immunfluoreszenz stellt nach wie vor den Goldstandard in der Detektion von antinukleären Antikörpern (ANA) dar, allerdings sind ANA nicht notwendigerweise mit Autoimmunerkrankungen assoziiert, ein Beispiel für nicht-pathogene ANA sind Anti-DFS70-
Ak. Dieses und Vieles mehr wird in kompetenter Weise von Mathias Wahle und Elisabeth Kling aus Augsburg diskutiert.

Septische Arthritis und Spondylodiszitis sind gottlob seltene, aber potentiell lebensgefährliche Infektionen mit zunehmender Inzidenz. Die septische Arthritis stellt den rheumatologischen Notfall im engeren Sinne dar; sie ist von hoher Morbidität und nicht unerheblicher Letalität gekennzeichnet. Patienten mit vorbestehenden Gelenkdestruktionen oder Immunsuppression sind besonders gefährdet, das gilt auch für die Spondylodiszitis. Vor Einleiten der Antibiotikatherapie sollte eine Erregersicherung und Leukozytenquantifizierung erfolgen. Die Zusammenfassung von Norma Jung, Köln gibt einen profunden Überblick über dieses Thema, vor allem hinsichtlich Diagnostik und Therapie.

Wie immer an dieser Stelle möchten wir Sie herzlich dazu einladen, uns Ihre Meinung zu den Beiträgen dieser Ausgabe, wie auch zu allen rheumatologischen Themen, die Sie für relevant oder für diskussionswert halten, zu schicken. Sie können in jedem Falle auf einen fairen Review mit entsprechendem Feedback vertrauen. Für Kritik, Hinweise, Zustimmung und für jeden anderen Beitrag sagen wir auch schon im Voraus Danke.

Herzlichst,

Ihr Burkhard Leeb

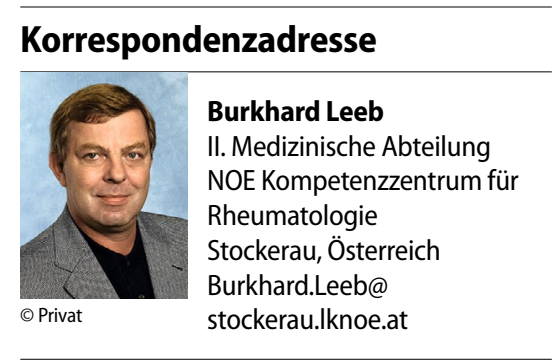

